\title{
An information model of a hydraulic press and its representation in the automated system
}

\author{
Evgeny Malygin ${ }^{1}$, Vladimir Mokrozub ${ }^{1, *}$, and Vladimir Nemtinov ${ }^{1}$ \\ ${ }^{1}$ Tambov State Technical University, 392000, Sovetskaya, 106, Tambov, Russia
}

\begin{abstract}
The paper describes the information model of a hydraulic press and its representation in the automated system. The proposed model makes it possible to design typical technical objects with minimal participation of a design engineer. The model allows determining the structure of a technical object, finding the dimensions of the elements and developing the working drawings.
\end{abstract}

\section{Introduction}

Technical object (TO) is a system that consists of the elements in a determined relationship with each other. TO and its elements have characteristics that ensure performance of functions for which is TO is designed.

Design of technical objects has two main directions: exploratory and standardized. The exploratory design results in the original design, which claims to obtain a patent. The standardized design results in the creation of TO assembled from standard elements. The paper deals with the development of an automated information system (AIS) intended for the design of hydraulic presses.

The AIS is based on the information model of the press (IMP) designed to produce documentation.

Currently, ISO 10303 standards regulate the way of presenting the information about the product, [1] proposes to use polychromatic sets to describe the properties of products, [2] provides methods of structural synthesis based on various graph models, [3] considers the presentation and design of product family, [4] describes methods of drawing up group specifications.

The above publications give little idea of how to make a description of the object in compliance with the technical specification (TS) so that it would be sufficient to produce the working draft of the TO with minimum human involvement and a full set of design drawings for parts and assembly units. Below we consider the information model of the press (IMP) and its representation in the information system that makes it possible to produce a working draft of the press according to the technical specification (TS).

\section{The structure of the information model of the press}

The initial data for the design of the press are the compressive force; the size of plates; the number of platens; the distance between the plates.
The AIS includes the following steps of obtaining embodiment (s) of the TO design: determining the TO structure (composition and element types), determining the dimensions of the elements, preparation of the working drawings.

According to the given steps the information model of a technical object (IM TO) can be described as follows:

$$
M=<E, M^{S}, M^{P}, M^{R}>\text {, Fig. } 1 .
$$

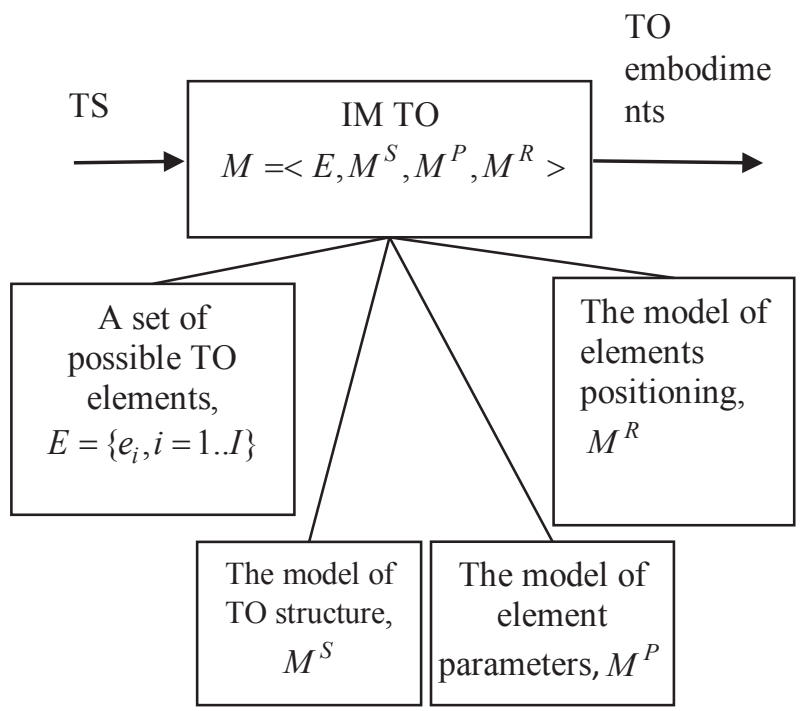

Fig. 1. The IM TO structure.

A set of possible TO elements. Each element of the TO $\left(e_{i}\right)$ has a set of properties (parameters) $P^{i}=\left\{p_{j}{ }^{i}, j=1 . . J^{i}\right\}$, and each property has a set of possible values $Z^{i j}=\left\{z_{k}^{i j}, k=1 . . K^{i j}\right\}$. The examples of element properties include the element type, geometry and specifications, material, etc.

\footnotetext{
Corresponding author: mokr@mail.gaps.tstu.ru
} 
The model of TO structure $\left(M^{S}\right)$ must provide for: - the selection of the elements which are required for the objet to perform its functions (e.g. if the object has the function of heating, it must have an element or elements providing heating);

- the selection of the type of element (e.g. if there is a heating plate and the operating temperature is more than $250 \mathrm{C}^{\circ}$, then the selected heating elements are RTDs ).

The basis of $M^{S}$ is AND-OR tree described in some detail, for example, in [1]. A fragment of AND-OR tree for the hydraulic press is shown in Fig. 2. AND-OR tree specifies possible structures for the TO. To find the structure and elements satisfying the TS it is necessary to have the rules to select these elements. These rules can be divided into two classes:

- the rules to determine the presence of the element in the TO. For example, if heating is required, the heating device must be present. These rules allow searching by the nodes labelled "AND", i.e. to form the structure of the TO.

- the rules to determine the type of the element. The rules allow searching by the nodes labelled "OR".

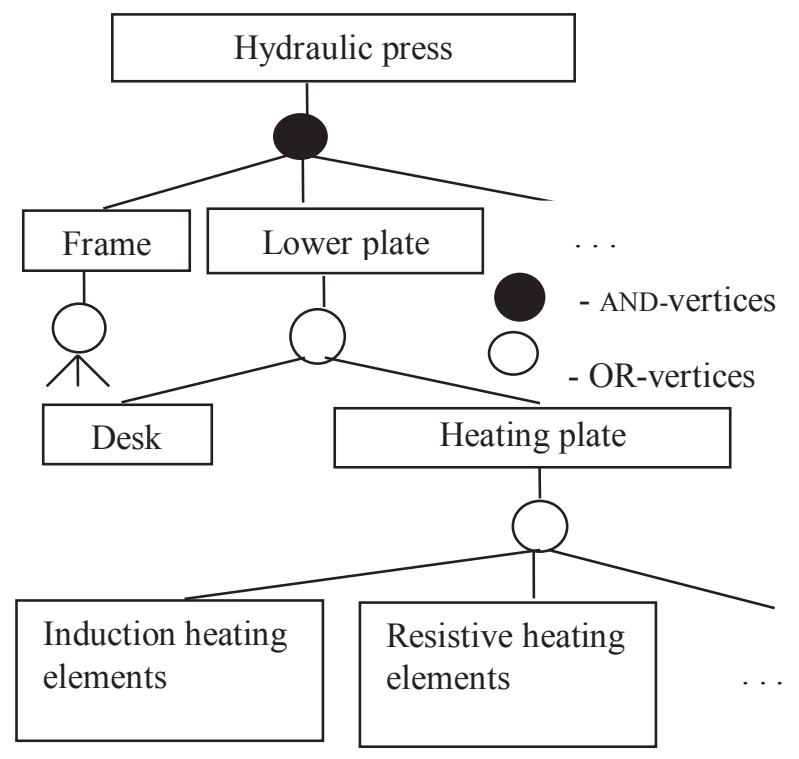

Fig. 2. A fragment of AND-OR tree for a hydralic press

The model of element parameters $M^{P}$ can be presented in the form $M^{P}=<M_{1}{ }^{P}, M_{2}{ }^{P}>$, where $M_{1}{ }^{P}$ is a set of standard dimensions of elements, $M_{2}{ }^{P}$ is a set of conditions (equations, rules), connecting element dimensions.

Standard elements of the TO in practice are represented in GOST, OST, STP in tables of dimensions (tables of bolts, flanges) which have one or more dimensions determining all the other (for example, for the hydraulic cylinder it is the diameter of a piston)

Dimensions of elements are the required parameters you need to know for the production of working drawings of parts and assembly units. Their number is great, but in practice (with the given structure of the $\mathrm{TO}$ ), and knowing the value of a small number of determining dimensions (DD) of the TO elements, one can calculate all the others. For example, for the hydraulic press, the DD is the length and the width of the heating plate and the number of platens of the press.

The dimensions of the TO elements can be divided into two types:

a) dimensions that depends on the dimensions of other elements of the TO, single parameters [1];

b) dimensions that depend on the other dimensions of the same element, unitary parameters [1].

The relationship of type b) dimensions of elements is represented as a table of standard dimensions. To represent the dependency of type a) one can use a variety of software tools, such as Mathcad.

Design engineers give preference to Mathcad as they can make and modify the expressions connecting the dimensions of type a).

The model of elements positioning. After identifying all parameters of the elements we can construct a 3D model of the TO (to assemble the elements), i.e. clearly define the position of elements relative to each other. Modern graphic editors allow building parametric 3D models of assemblies. However, their practical use in the design of a new TO is difficult, because you first need to build a lot of options of parametric 3D models, which are determined by ANDOR tree. It is preferable to make a mathematical description of the designed object, which will allow for a particular kind of structure to build its 3D model automatically. This description is called a model of elements positioning.

The formal presentation of the positioning model is as follows:

$$
M^{R}=<E^{3 D}, S, Y^{R}>,
$$

where $E^{3 D}$ is a set of $3 \mathrm{D}$ models elements; $S$ is a register of types of interfaces between the basic axes, edges and faces of the elements; $Y^{R}$ is the rules governing the interface between basic geometrical parameters of elements.

Any solid-state geometric object is characterized by a plurality of axes, edges, surfaces, which can be called the basic geometric elements (BGEs). The BGE interface types may be as follows:

$s_{1}$ is parallelism (or concurrency) of planes or axes;

$s_{2}$ is concentricity;

$s_{3}$ is intersection angle relative to each other (including perpendicularity);

$s_{4}$ is tapping;

$s_{5}$ is symmetry, etc.

When positioning the TO elements in space, firstly, we must identify the BGEs (axis edges, edges) of each element of the TO. Secondly, we must define the interfaces between the BGEs of the connected elements of the TO. By doing this, we obtain:

$$
Y^{R}=\bigcap_{t}\left(S_{k}{ }^{t}, p_{i n}, p_{j m}, x_{t}\right),
$$


where $t$ is the interface number, $S_{k}{ }^{t}$ is the interface type ( $k=1$ is parallelism, $k=2$ is concentricity, etc.), $p_{i n}$ is the $n$-th BGE of the $i$-th element of the TO, $p_{j m}$ is the $m$-th BRE of the $j$-th element of the TO, $x_{t}$ is the parameter characterizing the interface $t$ (the angle at the intersection of elements, the distance between the planes in the case of parallelism, etc.)

\section{Representation of the information model in the relational database}

In a relational database, a set of possible elements of the TO $E=\left\{e_{i}, i=1 . . I\right\}$ can be realized by the technology OBJECT-PROPERTY-VALUE (Fig. 3)

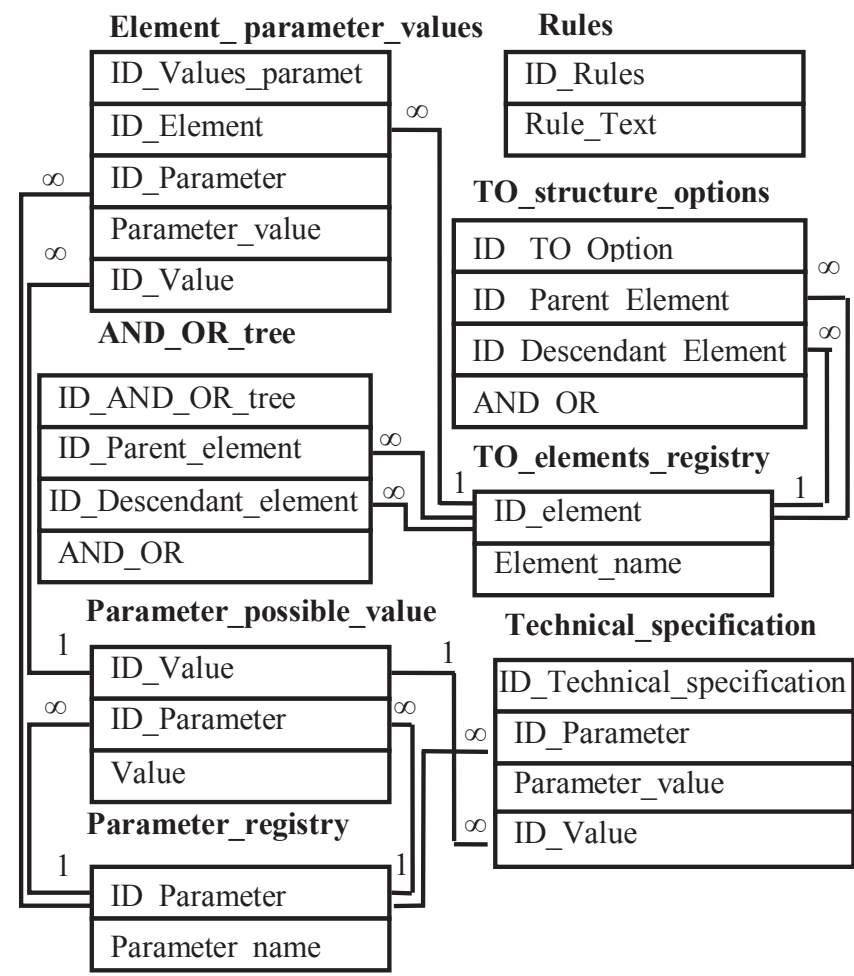

Fig. 3. The ER-diagram of a set of the TO possible elements and the model determining the TO structure

A set of the TO possible elements and their parameters is given in the Tables: "TO elements_registry", "Parameters_registry". "Parameter oossible values" and "Element parameters_values". For example, an element calle $\bar{d}$ "Hydraulic cylinder" is stored in the Table "TO_elements_registy", a parameter called "Piston diameter" is stored in the Table "Parameters registry" and the "Parameter value" of 400 is stored in the Table "Element_parameters values". The values of some parameters are a bounded set (e.g., the "Body_Material" can take on values from a finite list of steel). The lists of possible values of the parameters are in the Table 'Parameters possible values".

For representation of an AND-OR tree in a relational database, we use a hierarchical structure provided in [5]. In such a case, the Table "TO_elements_registry" contains both physical elements and element classes (e.g., bottom, unit flange, etc.). Obviously, classes can have parameters typical of the class elements. The Table "Technical_specification" (Fig. 3) contains the requirements to the TO specifies in the Table "Parameters registry". The Table "TO_structure_options" contains the result of combining the rules of selecting the elements from the Table "Rules" and the Tables "AND-OR_tree", "Element_ parameters_valuess" and "Technical_specification".

We consider one of the possible options for the rules in the Table "Rules". Assuming that for the parameter "Working temperature" ID_Parameter $=50$, for the element "Induction heating elements" ID_Element $=15$. Then the rule "If the operating temperature is less than $250^{\circ} \mathrm{C}$, the heating element must be induction type" in the form of a SQL statement is as follows:

SELECT ID Element, Name_element FROM Registry_Elements_TO PE ${ }^{-}$WHERE PE.ID_Element $=15$ AND EXISTS ( SELECT * FROM Technical_specification TS WHERE TS.ID_Paremeter $=50$ and TS.Value parameter $<250$ )

One can start checking rules in MS-SQL (T-SQL) using EXECUTE statement or SP_EXECUTESQL system procedure; the latter is preferable, as it allows for a dynamic substitution of parameters.

For representation of standard dimensions of elements in the relational database, one can use the following methods:

1) add dimensions of elements to the table "Parameters_registry" and fill in the Table "Element parameters_values" for each element (the Table "TO_elements_registry");

2) for each element of the TO create a table containing element dimensions and add a field with the name of the this table to the Table "TO_elements_registry";

3) create a wide table (for example, a table with a number of fields up to 100-200), which contains dimensions of all standard elements (one line corresponds to one physical standard element, one column corresponds to one dimension);

4) store information about dimensions in the form of the text string of the type "name_dimension =value_dimension", e.g., "D1 = 1000, D2 = 500, S = 8".

5) use combinations of the above listed methods.

All these methods have advantages and disadvantages. For example, when using method 2, we have lots of small tables; when using method 3, hard disk space is used inefficiently; when using method 4, it is necessary to make additional efforts to cut the actual value of the dimension out of the line.

The ER-Diagram of the positioning model is shown in Fig. 4.

To build the working drawings of the TO with minimal participation of the design engineer, one must have a library of parametric 3D models and 2D drawings of the elements. Legends of parametric dimensions must match the legends in the model of element parameters determination $M^{P}$. Here, we define the BGEs, which are used in the positioning model $M^{R}$. 
Dimensions in the model of element parameters determination can be designated as follows: unique_name_element.parametric_dimension.

\section{TO_elements_BGE}

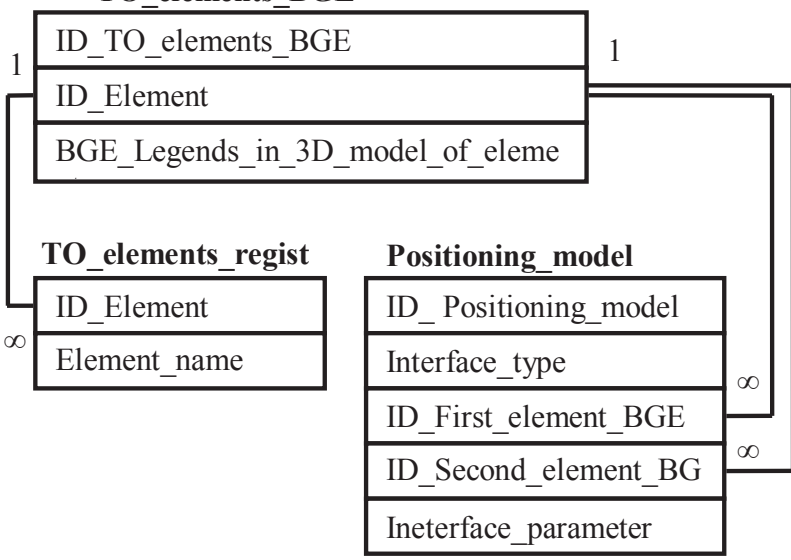

Fig. 4. The ER-diagram of the positioning model

Modern graphic editors (Compass, T-Flex, Inventor) have a programmer interface to create software for the following functions:

- reconstructing $3 \mathrm{D}$ models and $2 \mathrm{D}$ drawings of the TO elements from the library of standard elements using dimensions from $M^{P}$;

- constructing assembly units from the resulting elements of 3D models on the basis of the positioning model $M^{R}$.

\section{Results and discussion}

We considered the TO IM for the hydraulic press and its representation in the automated information system. The created IM consist of the registry of elements, the structure model, the parameters model, the TO positioning model. For representation of the IM in the automated system, we used the relational database. We described the structure of the relational database for the storage and processing of all elements of the IM.

The proposed structure of the IM and methods of its representation make it possible to create intelligent automated system for technical documentation generation with minimal human participation at different stages of the TO construction [6].

This work was financially supported by Ministry of Education and Science of the Russian Federation within the basic part (project 8.7082.2017/8.9).

\section{References}

1. V.V. Pavlov. Strukturnoe modelirovanie v CALStehnologijah [Structural modeling in CALStechnologies] (M.: Nauka, 2006) (in Russian)

2. G.S. Ivanova. Sposoby predstavlenija strukturnyh modelej [Methods of representation of structural models], Nauka i obrazovanie Jel. № FS 77-30569, № 1 (2007) (in Russian)

3. V.M. Tret'jakov, Osnovy metodiki proektirovanija semejstva izdelij [Fundamentals of product family design techniques], Avtomatizacija i sovremennye tehnologii. № 2, pp.25-33 (2004) (in Russian)

4. V.G. Mokrozub, Predstavlenie struktury izdelij v reljacionnoj baze dannyh [Representation of product structure in the relational database], Informacionnye tehnologii, №11, pp. 11-13 (2008) (in Russian)

5. V.G. Mokrozub, K.D. Manuilov, V.V. Gorshkov, T.S. Gorshkova, Chem. Petr. Eng., 51(9-10), pp. 613-618 (2015)

6. V.G. Mokrozub, V.A. Nemtinov, Chem. Petr. Eng., 51(7), pp. 487-492 (2015) 